\title{
Calcificación de los Procesos Estiloides: Sus Implicancias en Odontología. Presentación de un Caso
}

\author{
Ossification Styloid Processes: Implications in Dentistry. A Case Report.
}

Javier Elías Fernández Calderón ${ }^{1,2,3}$; María Elena Samar Romani \&ario Aníbal Sambrizzi Vicenzotti

FERNÁNDEZ, C. J. E.; SAMAR, R. M. E. \& SAMBRIZZI, V. M. A. Calcificación de los procesos estiloides: Sus implicancias en odontología. Presentación de un caso. Int. J. Odontostomat., 15(1):82-87, 2021.

RESUMEN: El proceso estiloides es una delgada prominencia ósea cónica localizada en la superficie inferior del hueso temporal. Su longitud normal es de 20 a $25 \mathrm{~mm}$, siendo mayor en los hombres. En ocasiones se produce su elongación o deformación con calcificación del ligamento estilohioideo, acompañado de diversos signos y síntomas lo que da origen al llamado síndrome de Eagle. La presencia de dolor inespecífico oro-cérvico-facial y la palpación de los procesos estiloides en la fosa tonsilar proporcionan un diagnóstico presuntivo que deberá ser confirmado con estudios por imágenes como la telerradiografía lateral de cráneo y la ortopantomografía. Se presenta un caso de síndrome de Eagle en un paciente de sexo masculino referido al Servicio de Diagnóstico por Imágenes de la Facultad de Odontología de la Universidad Nacional de Córdoba. La tomografía computada constituye el estudio por imágenes de excelencia ante la presunción de este síndrome ya que los diferentes cortes tomográficos y la reconstrucción 3D nos permiten observar los procesos estiloides elongados de manera precisa e inequívoca. Este diagnóstico es de gran relevancia ya que dependiendo de la severidad de la sintomatología el tratamiento puede ser quirúrgico para evitar las graves y severas complicaciones que a veces suele ocasionar.

PALABRAS CLAVE: odontología, anatomía, diagnóstico por imágenes.

\section{INTRODUCCIÓN}

El proceso estiloides (Processus styloideus) es una delgada prominencia ósea con forma de espolón o de colmillo localizada en la superficie inferior del hueso temporal desde donde se proyecta hacia abajo y adelante relacionándose en esta región anatómica con la vena yugular, la arteria carótida interna, el nervio vago y el nervio hipogloso. Se sitúa en el peñasco del temporal por delante del foramen estilomastoideo, debajo del meato auditivo externo y anterior al proceso mastoideo y la zona faríngea. Presta inserción a los ligamentos estilomandibular y estilohioideo y a los músculos estilogloso, estilofaríngeo y estilohioideo.

Embriológicamente deriva del cartílago del segundo arco faríngeo (Standring, 2005; Guzmán López et al., 2018). Proviene del llamado aparato hioideo que une el hueso hioides a la base del cráneo aunque en el adulto aparece unido al peñasco del temporal. Su longitud normal es de 20 a $25 \mathrm{~mm}$, siendo mayor en los hombres. El proceso estiloides se considera elongado cuando mide más de $30 \mathrm{~mm}$. En algunas ocasiones se produce una elongación o deformación de este proceso con calcificación del ligamento estilohioideo, acompañado de diversos signos y síntomas lo que da origen al llamado síndrome de Eagle (SE) (Kamal et al., 2014; El Kettani et al., 2015). Este síndrome fue descripto por primera vez por el otorrinolaringólogo americano Watt Weems Eagle en 1937 aunque ya lo menciona en $1652 \mathrm{el}$ anatomista italiano Pietro (Costa Ferreira et al., 2014; Kusunoki et al., 2016; Badhey et al., 2017).

El síndrome de Eagle también se denomina estilalgia, síndrome de la arteria carótida o síndrome estilo-carotídeo y presenta síntomas como disconfort faríngeo, dolor difuso y sordo localizado en la región

\footnotetext{
${ }^{1}$ Departamento de Biología Bucal. Facultad de Odontología. Universidad Nacional de Córdoba. Argentina.

2 Departamento de Patología Bucal. Facultad de Odontología. Universidad Nacional de Córdoba. Argentina.

${ }^{3}$ Área de Imagenología y Diagnóstico Digital. Facultad de Odontología. Universidad Nacional de Córdoba. Argentina.
} 
de cabeza y cuello, dolor de garganta, cefalea, otalgia, vértigo, hipersialia, disfagia, cambios en la fonación, dolor durante los movimientos cervicales y linguales y a veces produce compresión de la arteria carótida y de la vena yugular (Aldelaimi et al., 2016; Dermitas, et al., 2016; Madaleno et al., 2016; Benet-Muñoz et al., 2017; Li et al., 2019a; Omami, 2019). Su etiología exacta es desconocida y constituye una hiperplasia o una metaplasia reactiva asociada a una calcificación del tejido fibrocartilaginoso del ligamento estilohioideo como respuesta a un traumatismo o posterior a una tonsilectomía.

El síndrome de Eagle se observa en el 4 al 28 $\%$ de la población aunque sólo el $4 \%$ presenta sintomatología. Tiene mayor incidencia en personas mayores de 40 años, con predominio en el sexo femenino y casi siempre es bilateral siendo la calcificación completa unilateral muy poco frecuente, representando sólo del 7 al $10 \%$ de los casos (Piagkou et al., 2009; Hardy et al., 2014; Sowmya et al., 2016; Ata-Ali et al., 2017; Lei et al., 2017; Hettiarachchi et al., 2019).

Se debe realizar el diagnóstico diferencial con otras patologías que cursan con dolor orocervicofacial atípico como la neuralgia del trigémino, dolor orofacial atípico, dolor miofascial, carotidinia y disfunción de la articulación temporomandibular. Su diagnóstico se basa en la anamnesis, en la cuidadosa exploración clínica del paciente y principalmente en los estudios por imágenes. La presencia de dolor inespecífico orocérvico-facial y la palpación de los procesos estiloides en la fosa tonsilar proporcionan un diagnóstico presuntivo que deberá ser confirmado con estudios por imágenes como la telerradiografía lateral de cráneo y la ortopantomografía (OPT) que confirman la elongación de los procesos estiloides.

Los vertiginosos adelantos tecnológicos en informática y en las telecomunicaciones han aportado grandes avances al radiodiagnóstico odontológico. El surgimiento de la radiología digital y sus tecnologías de última generación nos han permitido disponer de equipos para radiología extraoral y tomografía computada cone beam (TCCB) que cuentan con software de fácil manejo y gran versatilidad que permiten realizar y trabajar con cortes y renderizados 3D de los huesos del área buco-maxilo-facial. Existen además monitores en escala de grises de alta resolución que permiten visualizar estas imágenes con alta calidad y definición (Eisenberg \& Amorosa, 1995; Dunn, 2001; García \& Ortega, 2002; Waites \& Drage, 2013).
La TCCB es precisamente una de los métodos que revolucionó el diagnóstico por imágenes en odontoestomatología. Esta técnica permite obtener además de cortes axiales, sagitales y coronales, cortes panorámicos y transversales de gran importancia en las distintas especialidades de la Odontología. Se obtienen cortes muy finos con vóxeles isotrópicos de $600 \mu \mathrm{m}$ hasta $75 \mu \mathrm{m}$, con una separación entre cortes también de hasta $75 \mu \mathrm{m}$ de distancia. En un ciclo de 18 segundos de exploración, con un campo de visión de 40 x $50 \mathrm{~mm}$ de diámetro y un tamaño del vóxel de $150 \mu \mathrm{m}$, el paciente recibe una dosis efectiva de radiación de tan solo 14,4 $\mu$ Sv (Fernández, 2016).

Por consiguiente, la TCCB proporciona niveles muy bajos de dosis de radiación para el paciente de acuerdo al principio ALARA es decir tan bajo como sea razonablemente posible (Waites \& Drage). La TCCB con reconstrucción 3D constituye en la actualidad el algoritmo de estudio por excelencia para diagnosticar el síndrome de Eagle ya que permite observar con precisión los procesos estiloides y las estructuras anatómicas adyacentes lo que además es de gran valor para una exitosa planificación quirúrgica (Khan et al., 2018).

\section{REPORTE DEL CASO}

Se presenta el caso de un paciente de sexo masculino, de 55 años de edad, de raza blanca, derivado al Servicio de Diagnóstico por Imágenes de la Facultad de Odontología de la Universidad Nacional de Córdoba para estudio preimplantológico. Se realizó una ortopantomografía (OPT) digital con $68 \mathrm{Kv}$ y $10 \mathrm{~mA}$ y una tomografía computada cone beam TCCB con 90 $\mathrm{K} v$ y $10 \mathrm{~mA}$, con el equipo promax-3D plus (Planmeca oy, Finlandia). Las imágenes obtenidas con ambos métodos de exploración fueron analizadas con las herramientas del software Romexis 4.4.0.R. Al analizar las imágenes de los estudios realizados se observaron los procesos estiloides anormalmente elongados por lo que se procedió a su estudio y análisis exhaustivo. Las medidas de los procesos estiloides se tomaron en las ortopantomografías teniendo en cuenta como punto de partida la parte más baja del hueso temporal donde se encuentra el hueso timpánico hasta el extremo caudal de la calcificación (Jung et al., 2004). Se tipificó la elongación de los procesos estiloides según la clasificación radiográfica de Langlais en clase I (elongación sin interrupción), clase II (unión del proceso estiloides con el ligamento 
estilohioideo calcificado en una pseudoarticulación) y clase III (calcificación que se presenta como segmentos separados) (Langlais et al., 1986; Magat \& Ozcan, 2017).

En la OPT se observó que los procesos estiloides se extendían como una gruesa franja radioopaca hasta las proximidades del ángulo de la mandíbula, con aspecto de pseudoarticulación (tipo II de Langlais) y con una longitud de $55,01 \mathrm{~mm}$ la derecha y $51,40 \mathrm{~mm}$ la izquierda (Figs. 1 y 2 ). Para la TCCB se empleó un campo de visión de $80 \mathrm{~mm}$ por $80 \mathrm{~mm}$, con un tamaño de voxel isotrópico de $200 \mu \mathrm{m}(0,2 \mathrm{~mm})$. Las imágenes obtenidas fueron visualizadas y anali- zadas con el software Romexis 4.4.0.R. Se analizaron cortes (axiales, frontales, sagitales, panorámicos y oblicuos) de $0,5 \mathrm{~mm}$ de espesor separados cada 0,5 $\mathrm{mm}$ y se realizó la renderización o reconstrucción del volumen 3D. En los diferentes cortes se observaron imágenes hiperdensas de hueso cortical. Al modificar la orientación de los cortes coronales se observó con mayor claridad la extensión de los procesos estiloides en los cortes sagitales (Fig. 3). En las imágenes tomográficas 3D se observaron los procesos estiloides elongados extendiéndose hasta el gognion o ángulo mandibular (Fig. 4). Al ser consultado en relación a alguna sintomatología específica, el paciente refirió dolores cervicales y cefaleas frecuentes.

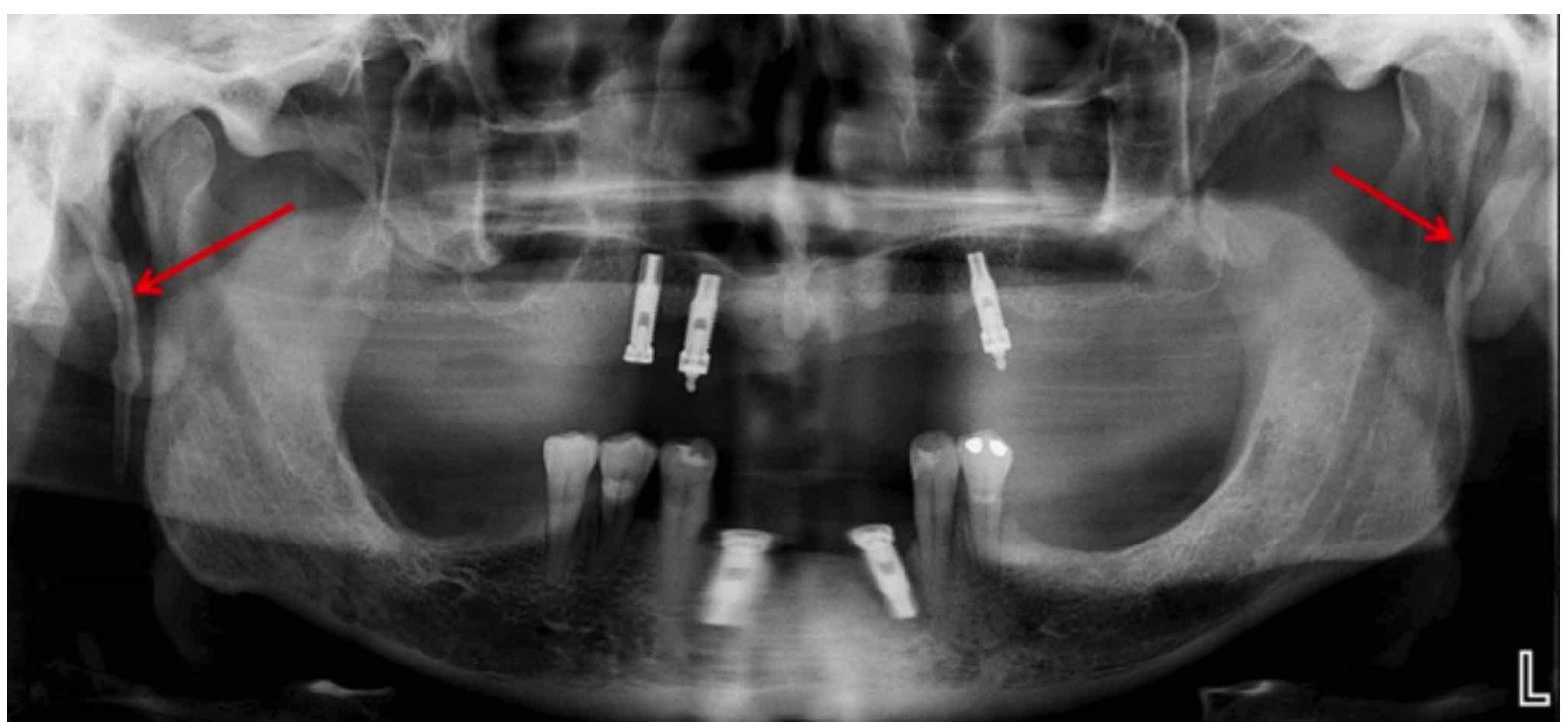

Fig. 1. Procesos estiloides elongados con aspecto de pseudoarticulación (tipo II de Langlais).

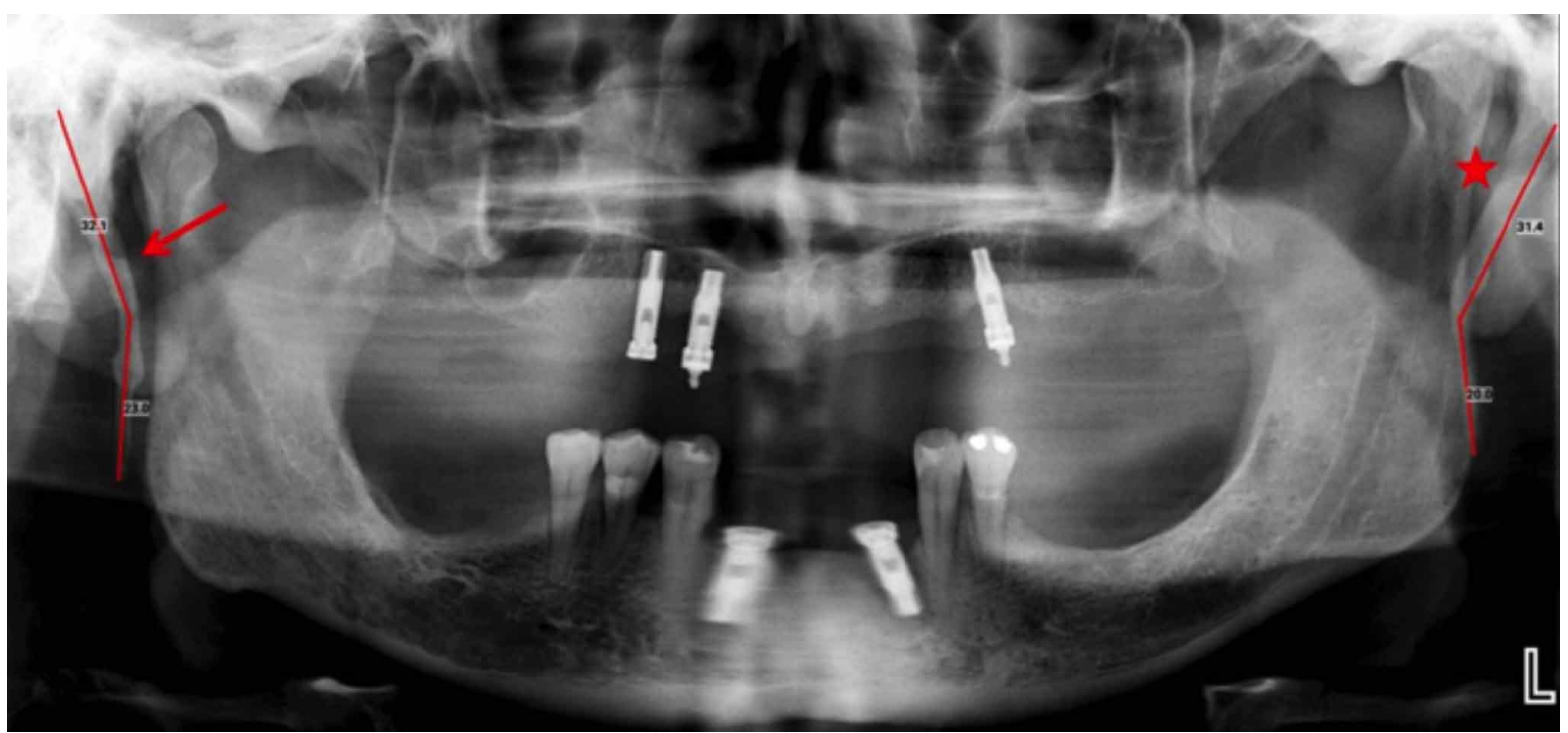

Fig. 2. Proceso estiloides derecho con una longitud de 55,01 $\mathrm{mm}$ (flecha) y proceso estiloides izquierdo con 51,40 $\mathrm{mm}$ de longitud (estrella). 

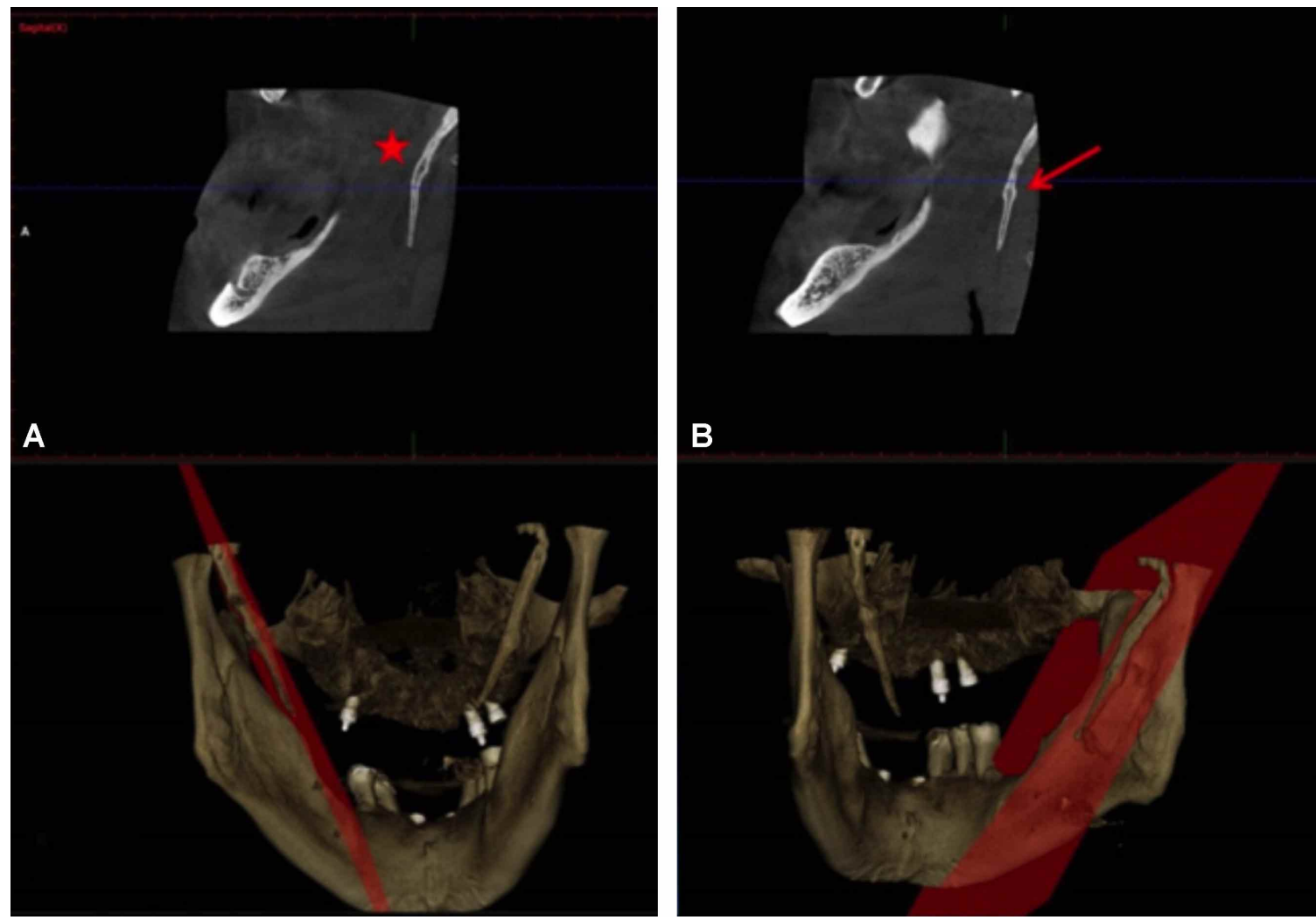

Fig. 3. Modificación de la orientación del plano sagital para observar los procesos estiloides elongados. a. Lado izquierdo (estrella). b. Lado derecho (flecha).

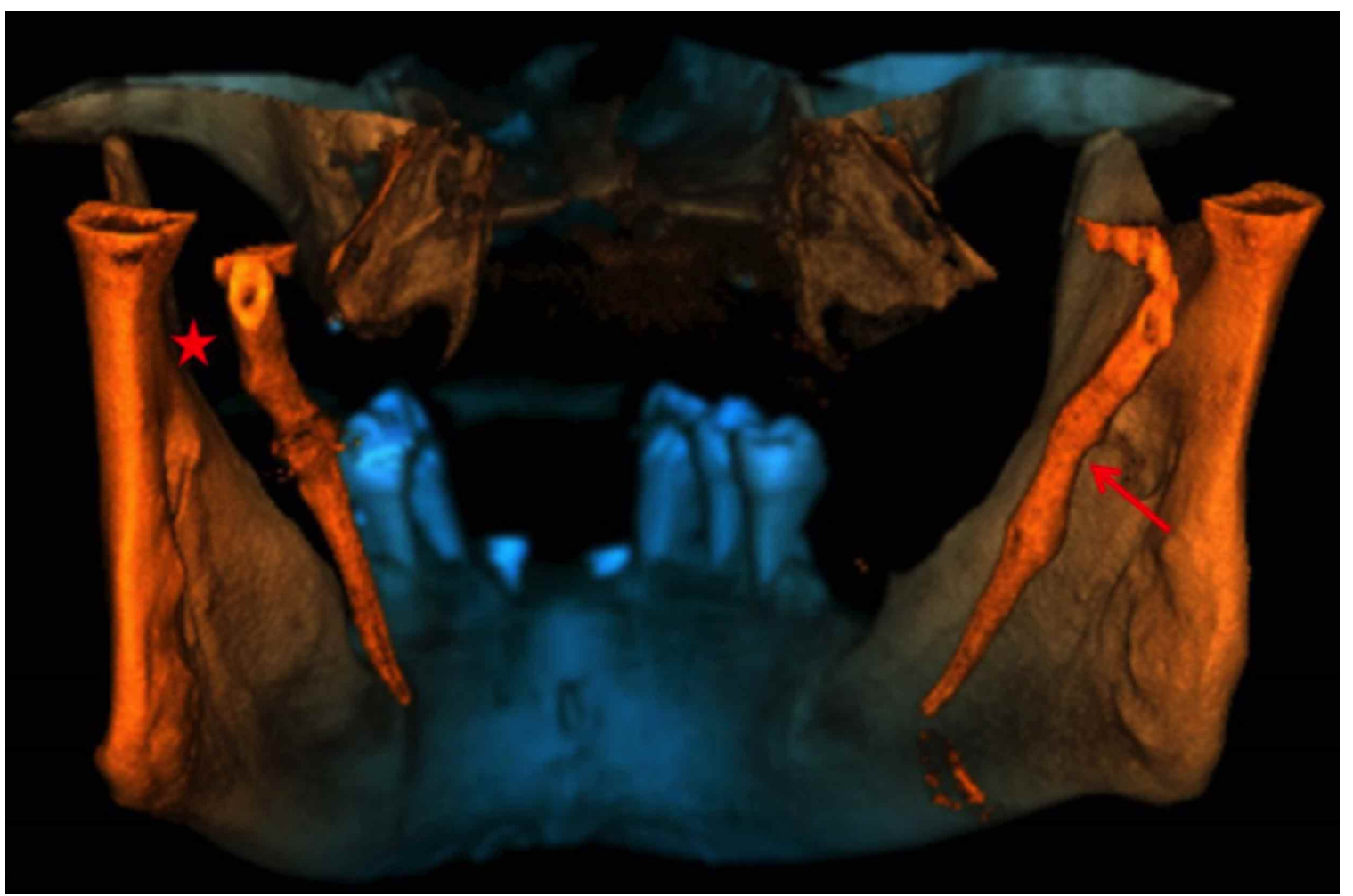

Fig. 4. Reconstrucción 3D. Vista posterior: Proceso estiloides izquierdo (estrella). Proceso estiloides derecho (flecha). 


\section{DISCUSIÓN}

En nuestro caso llamó la atención al realizar el análisis de las imágenes de la OPT y la TCCB la marcada elongación de los procesos estiloides, lo que constituye en la mayoría de los casos un infrecuente hallazgo radiológico que se observa en ortopantomografías que son estudios de rutina solicitados exclusivamente en Odontología.

El síndrome de Eagle es una patología poco frecuente que se caracteriza por presentar procesos estiloides de longitud anormalmente extensa, generalmente asintomática aunque en ocasiones puede manifestarse con una serie de signos y síntomas inespecíficos en el área orocervicofacial (Chang et al., 2015; Usseglio et al., 2016; Soylu et al., 2017). En raros casos puede ocasionar alteraciones severas como compresión de la arteria carótida o fracturas de stents carotídeos (Smoot et al., 2017; Qureshi et al., 2019). En la literatura se relatan tres casos de fallecimiento por fallo cardíaco debido a la compresión de los senos carotideos (Kumar, 2013; Clement \& Barrios, 2014; Gupta et al., 2017). Ante el diagnóstico de procesos estiloides elongados o de más de $30 \mathrm{~mm}$ de extensión, se deberá realizar una exhaustiva anamnesis y exploración clínica para un correcto diagnóstico y abordaje multidisciplinario.

En conclusión, siendo el SE una entidad nosológica que solo presenta síntomas en el $4 \%$ de los casos y los procesos estiloides reparos anatómicospoco observados por los odontólogos, se deberá profundizar la observación y el análisis de estas estructuras anatómicas en las OPT. Además el odontólogo será probablemente el primero en observar esta patología y deberá encaminar los estudios clínicos para su diagnóstico por lo cual debe conocer la existencia de este síndrome y saber cuáles son sus principales síntomas y signos clínicos y radiográficos. En determinados casos se deberán solicitar estudios más específicos como la TCCB que resultó ser el "gold standard" para el diagnóstico de este síndrome.

El diagnóstico temprano y preciso de este síndrome por parte del odontólogo permitirá realizar un abordaje preventivo y la derivación de los pacientes a un servicio de cabeza y cuello, ya que las severas complicaciones que a veces puede ocasionar son indicativas de un tratamiento quirúrgico (Kumai et al., 2016; Li et al., 2019b).
FERNÁNDEZ, C. J. E.; SAMAR, R. M. E. \& SAMBRIZZI, V. M. A. Ossification styloid processes: Implications in dentistry. A case report. Int. J. Odontostomat., 15(1):82-87, 2021.

ABSTRACT: Styloid process is defined as a thin conical bony prominence located on the undersurface of the temporal bone. Its normal length is 20 to $25 \mathrm{~mm}$. Sometimes styloid process is elongated with ossification of stylohyoid ligament and when it is accompanied by a set of signs and symptoms it gives rise to the so called Eagle's syndrome. Nonspecific pain in the oral cervicofacial area and styloid process palpation in the tonsillar fossa provide a presumptive diagnosis that should be confirmed by imaging studies such as lateral skull teleradiography and orthopantomography that confirm the elongation of styloid processes. An Eagle syndrome case of a male patient referred to the Department of Radiology at the National University of Córdoba School of Dentistry is reported. The computed tomography is the most accurate imaging modality for Eagle's syndrome identification because through multiplanar reformatted images and three dimensional images allows to observe the elongated styloid processes in a precise and unequivocal way. This diagnosis is relevant since depending on the severity of the symptoms, surgical treatment may avoid serious and severe complications.

KEY WORDS: dentistry, anatomy, imaging diagnostic.

\section{REFERENCIAS BIBLIOGRÁFICAS}

Aldelaimi, T. N.; Boskani, S. W.; Ali, S. M. \& Mahmud, P. K. Eagle syndrome: an unusual cause limited mouth opening and surgical management. J. Craniofacial. Surg., 28(3):e214-6, 2016.

Ata-Ali, J.; Ata-Ali, F.; Melo, M.; Andrés-Teruel, J. C. \& Soto-Sarrion, C. Eagle syndrome compared with stylohyoid syndrome: complete ossification of the stylohyoid ligament and joint. Br. J. Oral Maxillofac. Surg., 55(2):218-9, 2017.

Badhey, A.; Jategaonkar, A.; Kovacs, A. J. A.; Kadakia, S.; De Deyn, P. P.; Ducic, Y.; Schantz, S. \& Shin, E. Eagle syndrome: a comprehensive review. Clin. Neurol. Neurosurg., 159:34-s8, 2017.

Benet-Muñoz, S.; Tacoronte-Pérez, L.; Fernández-Belda, R. \& Artazkoz-del Toro, J. J. Síndrome de Eagle como causa infrecuente de disfagia. Rev. Gastroenterol. Mex., 82(3):257-8, 2017.

Chang, C. A.; Lin, T.; Fung, K.; Sharma, M. \& Fraser, J. A. Isolated Horner syndrome from an elongated styloid process (Eagle Syndrome). J. Neuroophthalmol., 35(4):387-9, 2015.

Clement, R. \& Barrios, L. Eagle syndrome and sudden and unexpected death: forensic point of view about one case. $J$. Forensic Res., 5(3):1000230, 2014.

Costa Ferreira, P.; Mendanha, M.; Frada, T.; Carvalho, J.; Silva, A. \& Amarante, J. Eagle syndrome. J. Craniofac. Surg., 25(1):e84-6, 2014.

Dermitas, , H.; Kayan, M.; Koyuncuog`lu, H. R.; Çelik, A.O.; Kara, M. \& S, engeze, N. Eagle syndrome causing vascular compression with cervical rotation: case report. Pol. J. Radiol., 81:277-80, 2016. 
Dunn, P. Wilhelm Conrad Röentgen (1845-1923), the discovery of $x$ rays and perinatal diagnosis. Arch. Dis. Child Fetal Neonatal Ed., 84(2):F138-9, 2001.

Eisenberg, R. \& Amorosa, J. K. The discoverer of x-rays: Wilhelm Conrad Roentgen. N. J. Med., 92(11):723-4, 1995.

El Kettani, N. E. C.; Benchaaboun, H.; El Hassani, M. R. \& Jiddane, M. Eagle's syndrome]. Presse Med., 44(5):560-1, 2015.

Fernández, J. E. Foramen mentoniano accesorio: presentación de un caso y revisión de la bibliografia. Rev. Argent. Anat. Clin., 8(3):151-6, 2016.

García, M. C. \& Ortega, T. D. Avances tecnológicos: la radiología que viene. Rev. Med. Chile, 130(6):699-703, 2002.

Gupta, A.; Aggrawal, A. \& Setia, P. A rare fatality due to calcified stylohyoid ligament (Eagle syndrome). Med. Leg. J., 85(2):1034, 2017.

Guzmán López, S.; Elizondo-Omaña, R. E; Bañuelos Rizo, M. \& Villarreal Silva E. E. Anatomía Humana: Manual de Prácticas Basadas en el Razonamiento Clínico. 2a ed. Buenos Aires, Médica Panamericana, 2018.

Hardy, H.; Guichard, B.; Eliezer, M.; Choussy, O.; Péron, J. M. \& Trost, O. Unilateral complete articulated ossification of the stylohyoid apparatus: case report and review of the literature. Surg. Radiol. Anat., 36(9):941-5, 2014.

Hettiarachchi, P. V. K. S.; Jayasinghe, R. M.; Fonseka, M. C.; Jayasinghe, R. D. \& Nanayakkara, C. D. Evaluation of the styloid process in a Sri Lankan population using digital panoramic radiographs. J. Oral Biol. Craniofac. Res., 9(1):73-6, 2019.

Jung, T.; Tschernitschek, H.; Hippen, H.; Schneider, B. \& Borchers, L. Elongated styloid process: when is it really elongated? Dentomaxillofac. Radiol., 33(2):119-24, 2004.

Kamal, A.; Nazir, R.; Usman, M.; Salam, B. \& Sana, F. Eagle syndrome; radiological evaluation and management. J. Pak. Med. Assoc., 64(11):1315-7, 2014.

Khan, H. M.; Fraser, A. D.; Daws, S.; Thoppay, J. \& Mupparapu, M. Fractured styloid process masquerading as neck pain: Conebeam computed tomography investigation and review of the literature. Imaging Sci. Dent., 48(1):67-72, 2018.

Kumai, Y.; Hamasaki, T. \& Yumoto, E. Surgical management of Eagle's syndrome: an approach to shooting craniofacial pain. Eur. Arch. Otorhinolaryngol., 273(10):3421-7, 2016.

Kumar, P.; Rayamane, A. P. \& Subbaramaiah, M. Sudden death due to Eagle syndrome: a case report. Am. J. Forensic Med. Pathol., 34(3):231-3, 2013.

Kusunoki, T.; Homma, H.; Kidokoro, Y.; Yanai, A.; Fuyimaki, M. \& Ikeda, $K$. A case of a very elongated styloid process $8 \mathrm{~cm}$ in length with frequent throat pain for 10 years. Clin. Pract., 6:820, 2016.

Langlais, R. P.; Miles, D. A. \& Van Dis, M. L. Elongated and mineralized stylohyoid ligament complex: a proposed classification and report of a case of Eagle's syndrome. Oral Surg. Oral Med. Oral Pathol., 61(5):527-32, 1986.

Lei, J.; Yap, A. U.; Zhang, Y. \& Fu, K. Y. Unilateral complete articulated ossification and aberrant thickening of the stylohyoid chain. $J$. Oral Sci., 59(1):157-60, 2017.

Li, M.; Gao, X.; Rajah, G. B.; Liang, J.; Chen, J.; Yan, F.; Bao, Y.; Jiao, L.; Zang, H.; Ding, Y.; et al. Styloidectomy and venous stenting for treatment of styloid-induced internal jugular vein stenosis: a case report and literature review. World Neurosurg., 130:129-32, 2019a.

Li, M.; Sun, Y.; Chan, C. C.; Fan, C.; Ji, X. \& Meng, R. Internal jugular vein stenosis associated with elongated styloid process: five case reports and literature review. BMC Neurol., 4:19(1):112, $2019 \mathrm{~b}$.

Madaleno, J.; Fernandes, A. \& Silva, N. A cause for craniocervical pain. Eur. J. Intern. Med., 29:e1-2, 2016.

Magat, G. \& Ozcan, S. Evaluation of styloid process morphology and calcification types in both genders with different ages and dental status. J. Istanb. Univ. Fac. Dent., 51(2):29-36, 2017.
Omami, G. Retromandibular pain associated with Eagle Syndrome. Headache, 59(6):915-6, 2019.

Piagkou, M.; Anagnostopoulou, S.; Kouladouros, K. \& Piagkos, G. Eagle's syndrome: a review of the literature. Clin. Anat., 22(5):545-58, 2009.

Qureshi, S.; Farooq, M. U. \& Gorelick, P. B. Ischemic stroke secondary to stylocarotid variant of Eagle Syndrome. Neurohospitalist, 9(2):105-8, 2019.

Smoot, T. W.; Taha, A.; Tarlov, N. \& Riebe, B. Eagle syndrome: A case report of stylocarotid syndrome with internal carotid artery dissection. Interv. Neuroradiol., 23(4):433-6, 2017.

Sowmya, G. V.; Singh, M. P.; Manjunatha, B. S.; Nahar, P. \& Astekar, M. A case of unilateral atypical orofacial pain with Eagle's syndrome. J. Cancer Res. Ther., 12(4):1323, 2016.

Soylu, E.; Altan, A.; Sekerci, A. E. \& Akbulut, N. An asymptomatic and overelongated styloid process. Case Rep. Dent., 2017:7971595, 2017.

Standring, S. Gray's Anatomy. The Anatomical Basis of Clinical Practice. 39a ed. London, Elsevier Churchill Livingstone, 2005.

Usseglio, J.; Macian Montoro, F.; Martin, S.; Lerat, J.; Laloze, J.; Taibi, A. \& Brie, j. Transcient ischemic attack, a rare manifestation of Eagle syndrome. Rev. Stomatol. Chir. Maxillofac. Chir. Orale, 117(6):421-4, 2016.

Waites, E. \& Drage, N. Essentials of Dental Radiography and Radiology E-Book. 5a Ed. Toronto, Elsevier, 2013.

Dirección para correspondencia:

Javier Elías Fernández Calderón

Departamento de Biología Bucal

Facultad de Odontología

Universidad Nacional de Córdoba

Dirección: Miguel Ibiac 1955

Córdoba

ARGENTINA

E-mail: javdens555@hotmail.com 